\title{
Prospective Analysis of a Sterile, Semi-automated Tissue Biopsy Homogenization Method in the Diagnosis of Prosthetic Joint Infections
}

\author{
CHRISTIAN SUREN, NORBERT HARRASSER, FLORIAN POHLIG, INGO J. BANKE, \\ ULRICH LENZE, FLORIAN LENZE, CAROLIN KNEBEL, RÜDIGER VON EISENHART-ROTHE, \\ JOHANNES SCHAUWECKER and HEINRICH M.L. MÜHLHOFER
}

\author{
Department of Orthopedic Surgery, Klinikum rechts der Isar, Technical University Munich, Munich, Germany
}

\begin{abstract}
Background/Aim: Prosthetic joint infection (PJI) remains a serious complication of total joint arthroplasty. To effectively treat PJI, it is essential to identify the microorganism causing it and be able to combine correct surgical and anti-infective treatments. This cannot always be achieved with the currently employed diagnostic methods. The aim of this study was to evaluate a semi-automated tissue biopsy bead milling method (Ultra-TurrAX, Axonlab AG; Reichenbach, Germany) based on the hypothesis that the results are more sensitive for microbe detection and less prone to contamination. Materials and Methods: We included 35 consecutive patients undergoing 38 hip or knee arthroplasty revisions in this study. In addition to manually processed biopsies, we processed tissue specimens harvested intraoperatively using a semi-automated method. The sensitivity and specificity of both methods were calculated using MSIS criteria and sonication results as gold standards. Results: For total hip arthroplasty samples were evaluated separately based on MSIS criteria as the reference standard, Ultra-TurrAX processing yielded $81 \%$ (62-100\%) sensitivity and $100 \%$ specificity. Using sonication as the gold standard, a sensitivity of $80 \%(60-100 \%)$ and specificity of $80 \%$ (45$100 \%)$ were calculated. In total knee arthroplasty, UltraTurrAX processing yielded 27\% (1-54\%) sensitivity and 57\% (20-94\%) specificity when using MSIS criteria as the gold standard. Using sonication as the gold standard, a sensitivity
\end{abstract}

This article is freely accessible online.

Correspondence to: Dr. Heinrich M.L. Mühlhofer, Department of Orthopedic Surgery, Klinikum rechts der Isar, Isamaninger Str. 22, 81675 Munich, Germany. Tel: +49 8941405490, Fax: +49 8941404849, e-mail: Heinrich.muehlhofer@mri.tum.de

Key Words: Prosthetic joint infection, tissue biopsy arthroplasty, implant-associated infection. of $60 \%$ (17-100\%) and specificity of 77\% (54-100\%) were calculated. Conclusion: This is the first study to analyze bead mill processing in total hip and knee arthroplasty revisions in a consecutive patient series. The method's sensitivity was comparable to and its specificity superior to regular sample processing results reported in the literature with respect to hip arthroplasties and to both hip and knee arthroplasties collectively. With respect to total knee arthroplasties, the method fared worse in our collective, most likely due to the small number of patients in the sample. Integrating the method into the clinical workflow allowed for speedier and more efficient sample handling and processing. The theoretical advantage of a lower risk of contamination because of fewer manual processing steps is, in our opinion, valid.

Prosthetic joint infection (PJI) remains a serious complication of total joint arthroplasty, and is likely to become even more important in the coming decades with the projected increase in life expectancy, population age and the number of arthroplasties performed. PJI poses a challenge not only due to the complexity of its surgical and medical treatment but also due to the difficulties encountered in establishing the correct diagnosis.

Currently, detecting infectious agents in synovial fluid aspirates or tissue biopsies is the mainstay of diagnosing PJI $(1,2)$. However, the reported accuracy of synovial fluid analysis varies in the literature, likely due to difficulties in detecting sessile organisms attached to biofilms $(1,3,4)$. Growing microorganisms from tissue specimens collected for diagnostic purposes or during prosthesis exchange surgery is reported to lead to a higher detection rate (5).

To increase diagnostic accuracy and establish a common diagnostic standard, a set of diagnostic criteria was defined by the Musculoskeletal Infection Society (MSIS) $(6,7)$. However, these criteria do not necessitate a direct identification of the infectious agent and its susceptibility to certain antibiotics. Identifying the optimal anti-infective 
treatment to accompany a one- or multi-stage surgical exchange is, however, essential for overall success.

Consequently, we believe that improving tissue biopsy yields is indispensable to achieving higher detection rates and detection accuracy in diagnosing PJI.

Toward this end, a sterile, semi-automated tissue homogenization method using magnetic beads has been introduced $(8,9)$. As opposed to widely used manual trituration, the tissue specimen is transferred directly into a sealed, sterile vessel in the operating room. In the microbiology laboratory, the tissue, still in the sterile vessel, is then milled via the acceleration of metal beads also contained in the vessel in a magnetic field without having to open the vessel. In theory, this technique decreases the risk of contamination by minimizing manipulation of the sample. Furthermore, it increases the number of pathogens prone to detection, whereas with commonly used methods for processing biopsies, there is a certain number of pathogens situated in inaccessible parts of the specimen.

Thus, our hypotheses for this study were: 1 . Sterile, semiautomated homogenization of tissue samples has superior sensitivity and specificity in the detection of PJI; 2 . This method reduces the contamination rate. The aims of this study were: 1 . To calculate the validity of microbiological detection after semi-automized homogenization, with MSIScriteria and sonication, each defined as gold standards. 2. To compare the contamination rates of the two sampling methods.

\section{Materials and Methods}

In this study, we included consecutive patients of legal age to give informed consent who had any indication for one- or multi-stage exchange of a total hip or knee arthroplasty. Patients with acute PJIs (both early postoperative and late onset hematogenous) and patients who did not give informed consent, or were unable to for medical reasons, were excluded.

Preoperative diagnostic criteria. For each joint, a preoperative diagnostic evaluation for PJI was performed according to our institution's standards. Evaluations included a radiographic analysis for signs of loosening, serological assessment of the C-reactive protein (CRP) level, white blood cell (WBC) and neutrophil count, erythrocyte sedimentation rate (ESR) measurement, and synovial fluid aspirate culture. In cases with inconsistent findings for these assessments, our institution's protocol calls for arthroscopic sampling for a tissue culture and histological analysis (10).

One or multi-stage prosthesis exchange was performed depending on whether the preoperative diagnostic findings suggested an infection or other types of failure.

Postoperative diagnostic criteria. As per institutional standards, we collected 3-6 tissue samples intraoperatively, that were sent to the microbiology laboratory for regular microbiological workup and semi-automated homogenization. They were allocated to either workup method as evenly as seen fit by the operating surgeon.
Explanted prosthesis components were sonicated (11), and the sonication results were used as the gold standard for postoperative sample assessment. Biopsy results inconsistent with the sonication results were deemed to be due to contamination if they only occurred in a single sample from a joint, if the microorganism was cultivated solely after enrichment, and if the histological analysis of the synovia-like interface membrane (SLIM) was not suggestive of infection (12). However, if a positive biopsy met the necessary criteria for PJI based on MSIS standards, the case was considered a PJI.

Semi-automated homogenization. For sample storage and transport, irradiated plastic vials with a sample volume capacity of up to $15 \mathrm{ml}$, containing $2 \mathrm{ml}$ of water and 10 stainless-steel beads (UltraTurrAX, Axon Lab AG; Reichenbach, Germany), were used (Figure 1). Each sample was transferred into a vial, which was then sealed. For milling, the sealed vial was put in a dispersion device (UltraTurrAX Dispersion Device, Axon Lab AG; Reichenbach, Germany), which accelerated the beads magnetically for $3 \mathrm{~min}$ (Figure 2). For samples containing bone fragments or other tough tissues, the acceleration and duration can be adjusted. After homogenization, the sample can be used for standard microbiological culture and can be kept sealed and frozen for later analysis.

Data analysis. Binary parameters (MSIS criteria met vs. not met, sonication positive $v s$. negative, contamination $v s$. no contamination) were analyzed using chi-squared tests. We calculated the sensitivity and specificity, positive and negative predictive values and likelihood ratios for conventional tissue culturing and sterile homogenization, using sonication results and MSIS criteria as gold standards.

\section{Results}

Over a time period of 11 months (July 2015-May 2016), we included 35 (16 male, 19 female) patients with a total of 38 examined total hip and knee arthroplasties (20 hip, 18 knee arthroplasties) in this study. Average patient age was 70.3 (range $=36-88$ ) years.

Twenty-seven patients met the MSIS criteria for PJI, and 11 were classified as negative for PJI. Twenty patients had microorganisms recovered from their sonication fluid, and 18 patients did not.

Arthroscopy for the tissue sample workup before prosthesis exchange surgery was performed in 6 instances ( 3 hip, 3 knee arthroplasties). Two of these samples were classified as debris-induced (type 1) membranes, in one of which, the sonication result was positive and the MSIS criteria were negative. In the other, both the sonication result and MSIS criteria were negative. One histological sample was judged to show neither infection nor debris-induced synovitis (type 4 membrane), and both the sonication result and MSIS criteria were negative.

Two arthroscopies showed purulence in the joints in instances of recurrent acute infection; therefore, after collecting samples for microbiology workup, no further histological samples were collected, and the prostheses were 


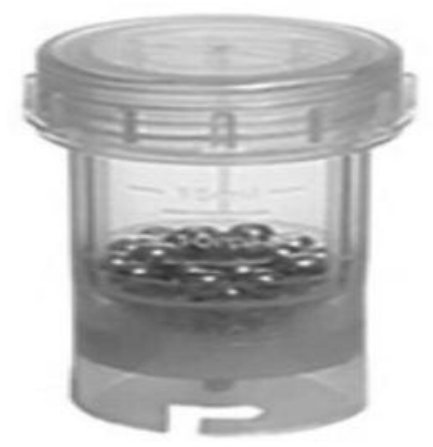

Figure 1. Standard vial containing $10 \mathrm{ml}$ of sterile saline and 10 magnetic beads. Image courtesy of Axonlab AG; Reichenbach, Germany.

explanted in the same session.

Semi-automized homogenization in total hip arthroplasty. In the 20 total hip arthroplasties examined, the combination of regular tissue-sample workup and homogenization with the MSIS criteria as the reference standard yielded a sensitivity of $88 \%(71-100 \%)$ and a specificity of $75 \%(33-100 \%)$. Positive and negative predictive values for tissue biopsies overall were $93 \%(81-100 \%)$ and $60 \%(17-100 \%)$, respectively. The positive likelihood ratio was $3.5(-5.95-$ $1.06)$, and the negative, $0.17(-0.03-0.88)$.

With sonication results as the reference standard, the sensitivity and specificity of all tissue samples combined were $87 \%(69 \%-100 \%)$ and $60 \%(17-100 \%)$, respectively. The positive predictive value was $87 \%(69-100 \%)$, and the negative predictive value was $60 \%(17-100 \%)$. The positive likelihood ratio was 2.17 (0.84-35.31), and the negative, 0.22 (0-1.79).

Evaluated separately and with MSIS criteria as the reference standard, Ultra-TurrAX-processed samples yielded an $81 \%(62-100 \%)$ sensitivity and $100 \%$ specificity, a positive predictive value of $100 \%$ and a negative predictive value of $57 \%(20-94 \%)$, with a negative likelihood ratio of $0.19(0-0.38)$. Regular tissue samples yielded an $88 \%(71-100 \%)$ sensitivity, a $75 \%(33-100 \%)$ specificity, a positive predictive value of $93 \%$ (81-100\%), a negative predictive value of $60 \%(17-100 \%)$, a positive likelihood ratio of 3.5 (1.06-5.95) and a negative likelihood ratio of $0.17(0-0.88)$.

With sonication as the reference standard, a sensitivity of $80 \%(60-100 \%)$ and specificity of $80 \%(45-100 \%)$ were calculated for Ultra-TurrAX samples, along with positive and negative predictive values of $92 \%(78-100 \%)$ and $57 \%$ (2094\%), respectively, and positive and negative likelihood ratios of 4 (1.09-6.6) and $0.25(0-0.9)$, respectively.

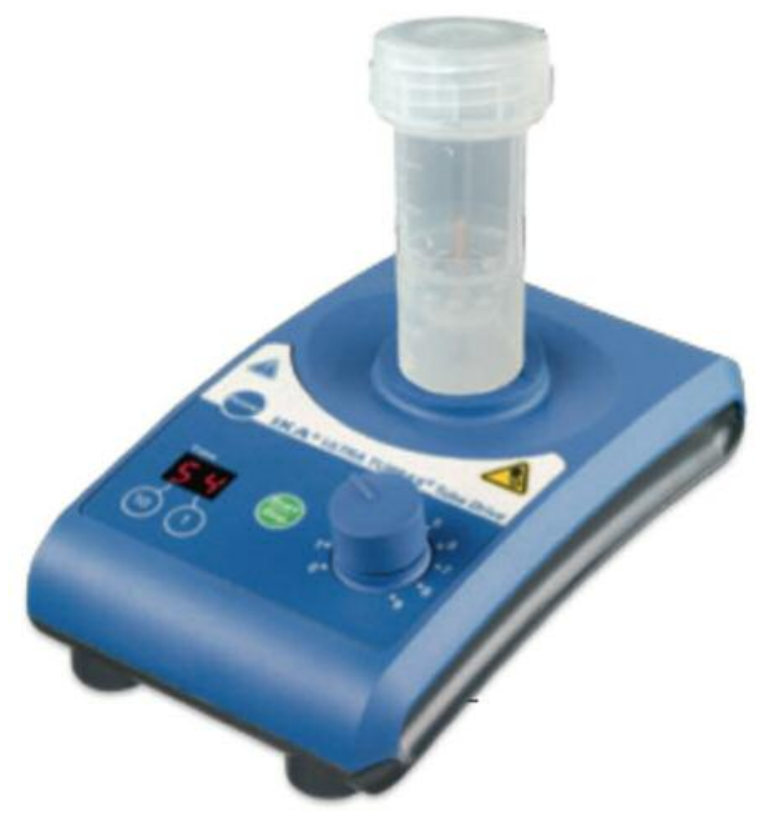

Figure 2. Dispersion device. Image courtesy of Axonlab AG; Reichenbach, Germany.

For regular samples, we calculated an $87 \%(69-100 \%)$ sensitivity, 60\% (17-100\%) specificity, 87\% (69-100\%) positive predictive value, and $60 \%$ (17-100\%) negative predictive value, with a positive likelihood ratio of 2.17 (0.84-35.31) and a negative ratio of 0.22 (0-1.79). For an overview of the results with MSIS criteria as a gold standard, see Table I. For an overview of the results with sonication as a gold standard, see Table II.

Semi-automated homogenization in total knee arthroplasty. In the 18 total knee arthroplasties examined, the combination of regular tissue-sample workup and homogenization with MSIS criteria as the reference standard yielded a sensitivity of $27 \%(1-54 \%)$ and a specificity of $57 \%(20-94 \%)$. The positive and negative predictive values for tissue biopsies overall were 50\% (10-90\%) and 33\% (7-60\%), respectively. The positive likelihood ratio was $0.64(0.01-8.65)$, and the negative, 1.27 (0.49-4.84).

With sonication results as the reference standard, the sensitivity and specificity of both methods combined were $60 \%(17-100 \%)$ and $77 \%(54-100 \%)$, respectively. The positive predictive value was $50 \%(10-90 \%)$, and the negative predictive value was $83 \%(62-100 \%)$. The positive likelihood ratio was 2.6 (0.37-5.93), and the negative, 0.52 (0-1.54).

Evaluated separately and with MSIS criteria as the reference standard, Ultra-TurrAX-samples yielded $27 \%$ (1-54\%) sensitivity and 57\% (20-94\%) specificity, a positive 
Table I. Results of Ultra-TurrAX and regular processing of tissue biopsies from total hip and knee arthroplasties with MSIS criteria for prosthetic joint infection as the gold standard.

\begin{tabular}{|c|c|c|c|c|c|c|c|c|}
\hline Joint & $\begin{array}{c}\text { Reference } \\
\text { standard }\end{array}$ & Test & Sensitivity & Specificity & $\begin{array}{l}\text { Positive } \\
\text { predictive } \\
\text { value }\end{array}$ & $\begin{array}{c}\text { Negative } \\
\text { predictive } \\
\text { value }\end{array}$ & $\begin{array}{c}\text { Positive } \\
\text { likelihood } \\
\text { ratio }\end{array}$ & $\begin{array}{c}\text { Negative } \\
\text { likelihood } \\
\text { ratio }\end{array}$ \\
\hline \multirow[t]{2}{*}{ Hip \& Knee } & \multirow[t]{2}{*}{ MSIS } & Ultra-TurrAX & $0.59(0.41-0.78)$ & $0.73(0.46-0.99)$ & $0.84(0.68-1.00)$ & $0.42(0.2-0.64)$ & $2.17(0.76-81.58)$ & $0.56(0.22-1.28)$ \\
\hline & & Tissue Culture & $0.8(0.62-0.98)$ & $0.89(0.74-1.00)$ & $0.89(0.74-1.00)$ & $0.8(0.62-0.98)$ & $7.2(0-2.44)$ & $0.23(0.02-0.5)$ \\
\hline \multirow[t]{2}{*}{ Hip } & \multirow[t]{2}{*}{ MSIS } & Ultra-TurrAX & $0.81(0.62-1.00)$ & 1 & 1 & $0.57(0.2-0.94)$ & - & $0.19(0-0.38)$ \\
\hline & & Tissue Culture & $0.88(0.71-1.00)$ & $0.75(0.33-1.00)$ & $0.93(0.81-1.00)$ & $0.6(0.17-1.00)$ & $3.5(1.06-5.95)$ & $0.17(0-0.88)$ \\
\hline \multirow[t]{2}{*}{ Knee } & \multirow[t]{2}{*}{ MSIS } & Ultra-TurrAX & $0.27(0.01-0.54)$ & $0.57(0.2-0.94)$ & $0.5(0.1-0.9)$ & $0.33(0.07-0.6)$ & $0.64(0.01-8.65)$ & $1.27(0.49-4.84)$ \\
\hline & & Tissue culture & $0.6(0.17-1.00)$ & 1 & 1 & $0.87(0.69-1.0)$ & - & 0.4 \\
\hline
\end{tabular}

Table II. Results of Ultra-TurrAX and regular processing of tissue biopsies from total hip and knee arthroplasties with results of sonication of the explanted prosthesis components as the gold standard.

\begin{tabular}{|c|c|c|c|c|c|c|c|c|}
\hline Joint & $\begin{array}{c}\text { Reference } \\
\text { standard }\end{array}$ & Test & Sensitivity & Specificity & $\begin{array}{l}\text { Positive } \\
\text { predictive } \\
\text { value }\end{array}$ & $\begin{array}{c}\text { Negative } \\
\text { predictive } \\
\text { value }\end{array}$ & $\begin{array}{c}\text { Positive } \\
\text { likelihood } \\
\text { ratio }\end{array}$ & $\begin{array}{c}\text { Negative } \\
\text { likelihood } \\
\text { ratio }\end{array}$ \\
\hline \multirow[t]{2}{*}{ Hip \& Knee } & Sonication & Ultra-TurrAX & $0.75(0.56-0.94$ & $0.78(0.59-0.97)$ & $0.79(0.61-0.97)$ & $0.74(0.54-0.93)$ & $3.38(1.35-31.16)$ & $0.32(0.06-0.75)$ \\
\hline & & Tissue Culture & $0.75(0.56-0.94$ & $0.88(0.73-1.0)$ & $0.88(0.73-1.0)$ & $0.75(0.56-0.94)$ & $6.38(2.07-26.46)$ & $0.28(0.06-0.6)$ \\
\hline \multirow[t]{2}{*}{ Hip } & Sonication & Ultra-TurrAX & $0.8(0.6-1.0)$ & $0.8(0.45-1.0)$ & $0.92(0.78-1.0)$ & $0.57(0.2-0.94)$ & $4(1.09-6.6)$ & $0.25(0-0.9)$ \\
\hline & & Tissue Culture & $0.87(0.69-1.0)$ & $0.6(0.17-1.0)$ & $0.87(0.69-1.0)$ & $0.6(0.17-1.0)$ & $2.17(0.84-35.31)$ & $0.22(0-1.79)$ \\
\hline \multirow[t]{2}{*}{ Knee } & Sonication & Ultra-TurrAX & $0.6(0.17-1.0)$ & $0.77(0.54-1.00)$ & $0.5(0.1-0.9)$ & $0.83(0.62-1.0)$ & $2.6(0.37-593)$ & $0.52(0-1.54)$ \\
\hline & & Tissue Culture & $0.6(0.17-1.0)$ & 1 & 1 & $0.87(0.69-1.0)$ & - & $0.4(0-0.83)$ \\
\hline
\end{tabular}

predictive value of $50 \%(10-90 \%)$ and a negative predictive value of $33 \%(7-60 \%)$, with a positive likelihood ratio of $0.64(0.01-8.65)$ and a negative likelihood ratio of 1.27 (0.49-4.84). Regular tissue samples yielded 60\% (17-100\%) sensitivity, $100 \%$ specificity, a positive predictive value of $100 \%$, a negative predictive value of $87 \%(69-100 \%)$, and a negative likelihood ratio of 0.4 .

With sonication as the reference standard, a sensitivity of $60 \%(17-100 \%)$ and specificity of $77 \%$ (54-100\%) were calculated for Ultra-TurrAX samples, along with positive and negative predictive values of $50 \%(10-90 \%)$ and $83 \%$ (62$100 \%)$, respectively. The positive and negative likelihood ratios were $2.6(0.37-5.93)$ and $0.52(0-1.54)$, respectively.

For regular samples, we calculated $60 \%$ (17-100\%) sensitivity, $100 \%$ specificity, a $100 \%$ positive predictive value, an $87 \%(69-100 \%)$ negative predictive value, and a negative likelihood ratio of $0.4(0-0.83)$. For an overview of the results with MSIS criteria as gold standard, see Table I. For an overview of the results with sonication as gold standard, see Table II.

Contaminations. There was a total of 6 patients with suspected contamination of 1 or more samples.
Two patients had two contaminated regular tissue samples each: in the first case, the MSIS criteria were positive for infection, there was a type 3 periprosthetic membrane, and sonication, as well as regular and UltraTurrax, processing of biopsies yielded Propionibacterium granulosum in microbiological culture. One regular biopsy, however, was positive for Staphylococcus saprophyticus and another for Micrococcus luteus.

The second patient did not meet the MSIS criteria, histology was not suggestive of an infection (type 1 membrane), and sonication- and UltraTurrax-processed biopsies were negative. In two regular tissue specimens, however, Staphylococcus epidermidis was detected.

Three patients had likely contaminations in one UltraTurrax-processed biopsy each: The first patient had positive MSIS criteria and histological results, and sonication and regular processing of one tissue specimen yielded Staphylococcus aureus. One UltraTurrax-processed sample showed Staphylococcus epidermidis growth.

The second patient did not exhibit positive MSIS criteria, there was a type 1 , debris-induced membrane, and regular tissue biopsies were negative. One UltraTurrax-processed sample showed Staphylococcus hominis growth. 


\section{Discussion}

In an experimental approach, bead mill tissue sample homogenization was superior to regular sample collecting and processing methods $(8,9)$. In theory, the described method has several advantages. First, it minimizes the number of manipulations in the sampling and evaluation process, which presumably reduces contamination rates. Second, homogenization theoretically enables an increased "bacteria yield" per sample as the homogenized tissue can be evenly distributed on culture media. In regular samples, microorganisms might persist in pouches or folds of tissue that is not thoroughly ground and be inaccessible. Third, in combination with reaching a consensus on tissue sample size, standardizing vessel size, the number of magnetic beads and milling time would present a further step towards a truly standardized approach for diagnosing PJI.

To our knowledge, there has only been one other study evaluating sterile semi-automated tissue homogenization in PJIs in a clinical setting (13). The authors reported a documentation rate for PJI of $83.7 \%$, with a contamination rate of $8.7 \%$. The patient collective consisted of 92 patients with known PJI. Our own data for hip and knee arthroplasties combined and for hip arthroplasties alone are comparable to those published by Roux et al., although our patient series did not consist only of patients with known PJI (13).

In comparison with other reported validations of tissue sampling, our data for knee arthroplasties show worse results, in respect to both regular and Ultra-TurrAX sampling methods $(11,14)$. The calculated validities for both methods in hip and knee arthroplasty and in hip arthroplasty infections alone, however, are comparable to previously published data $(2,11,15-17)$.

Our first hypothesis, that semi-automized homogenization has superior sensitivity and specificity, was not supported. In total hip arthroplasties, the method had slightly lower sensitivity rates compared with the results of regular tissue sampling. However, specificity was considerably higher (100\% vs. $75 \%$ with MSIS criteria as the gold standard, $80 \%$ vs. $60 \%$ with sonication results as the gold standard). The opposite was seen in total knee arthroplasties: here, the sensitivity and specificity achieved via Ultra-TurrAXprocessing were invariably lower compared with the results of the regular sampling and processing method.

This inconsistency was not seen in a study by Schäfer $e t$ al., who were able to analyze hip and knee arthroplasty data as a common collective for a lack of differences (18). Our observations might be due to the small number of patients and the resulting lack of power of this study. Subdividing patients into hip and knee arthroplasty groups could have further accentuated the diverging trends in the results. A more homogenous patient collective (including only patients with known PJI) might have yielded more consistent data.
However, we used the trial Ultra-TurrAX vials made available to us in every clinical situation in which we collect tissue samples. While this choice of patient collective reduces the comparability of the results, one of our main interests was how well the method could be integrated into our workflow. In the personal opinion of the surgeons, OR personnel and laboratory technicians in our Microbiology Institute who were involved in the trial, sample handling and workflow using Ultra-TurrAX vials was speedier, more efficient and less prone to mistakes.

While the small number of patients and of documented contaminations prevents any statistical deductions, the number of contaminations was twice as high in regularly processed specimens. Whether this is proof of theoretical and anecdotal advantages of the method remains to be studied with a broader approach.

\section{Conflicts of Interest}

The Authors declare that there are no conflicts of interest.

\section{References}

1 Fink B, Schafer P and Frommelt L: (Logistic requirements and biopsy of periprosthetic infections: what should be taken into consideration?). Orthopade 41(1): 15-19, 2012.

2 Zimmerli W, Trampuz A and Ochsner PE: Prosthetic-joint infections. N Engl J Med 351(16): 1645-1654, 2004.

3 Saleh KJ, Clark CR, Sharkey PF, Goldberg VM, Rand JA and Brown GA: Modes of failure and preoperative evaluation. J Bone Joint Surg Am 85-A(Suppl 1): S21-25, 2003.

4 Schafer P, Fink B, Sandow D, Margull A, Berger I and Frommelt L:Prolonged bacterial culture to identify late periprosthetic joint infection: a promising strategy. Clin Infect Dis 47(11): 1403$1409,2008$.

5 Aggarwal VK, Higuera C, Deirmengian G, Parvizi J and Austin MS: Swab cultures are not as effective as tissue cultures for diagnosis of periprosthetic joint infection. Clin Orthop Relat Res 471(10): 3196-3203, 2013.

6 Workgroup Convened by the Musculoskeletal Infection S: New definition for periprosthetic joint infection. J Arthroplasty 26(8): 1136-1138, 2011.

7 Parvizi J, Zmistowski B, Berbari EF, Bauer TW, Springer BD, Della Valle CJ, Garvin KL, Mont MA, Wongworawat MD and Zalavras CG: New definition for periprosthetic joint infection: from the Workgroup of the Musculoskeletal Infection Society. Clin Orthop Relat Res 469(11): 2992-2994, 2011.

8 Warnke P, Redanz S, Zaatreh S and Podbielski A: Augmented recovery of microorganisms from swabs by homogenization: a novel standardizable high-throughput approach. Diagnostic MicrobiolInfect Dis 84(1): 16-18, 2016.

9 Redanz S, Podbielski A and Warnke P: Improved microbiological diagnostic due to utilization of a highthroughput homogenizer for routine tissue processing. Diagn MicrobiolInfect Dis 82(3): 189-193, 2015.

10 Muhlhofer HM, Gollwitzer H, Lenze F, Feihl S, Pohlig F, von Eisenhart-Rothe R and Schauwecker J: (Periprosthetic infections 
of the hip joint: Clinical approach). Orthopade 44(5): 357-365, 2015.

11 Trampuz A, Piper KE, Jacobson MJ, Hanssen AD, Unni KK, Osmon DR, Mandrekar JN, Cockerill FR, Steckelberg JM, Greenleaf JF and Patel R:Sonication of removed hip and knee prostheses for diagnosis of infection. N Engl J Med 357(7): 654663, 2007.

12 Morawietz L, Classen RA, Schroder JH, Dynybil C, Perka C, Skwara A, Neidel J, Gehrke T, Frommelt L, Hansen T, Otto M, Barden B, Aigner T, Stiehl P, Schubert T, Meyer-Scholten C, Konig A, Strobel P, Rader CP, Kirschner S, Lintner F, Ruther W, Bos I, Hendrich C, Kriegsmann J and Krenn V: Proposal for a histopathological consensus classification of the periprosthetic interface membrane. J Clin Pathol 59(6): 591-597, 2006.

13 Roux AL, Sivadon-Tardy V, Bauer T, Lortat-Jacob A, Herrmann JL, Gaillard JL and Rottman M: Diagnosis of prosthetic joint infection by beadmill processing of a periprosthetic specimen. Clin Microbiol Infect 17(3): 447-450, 2011.

14 Fink B, Makowiak C, Fuerst M, Berger I, Schafer P and Frommelt L: The value of synovial biopsy, joint aspiration and C-reactive protein in the diagnosis of late peri-prosthetic infection of total knee replacements. J Bone Joint Surg Br 90(7): 874-878, 2008.
15 Peel TN, Buising KL and Choong PF: Prosthetic joint infection: challenges of diagnosis and treatment. ANZ J Surg 81(1-2): 3239,2011

16 Atkins BL, Athanasou N, Deeks JJ, Crook DWM, Simpson H, Peto TEA, McLardy-Smith P and Berendt AR: Prospective Evaluation of Criteria for Microbiological Diagnosis of Prosthetic-Joint Infection at Revision Arthroplasty. J Clin Microbiol 36(10): 2932-2939, 1998.

17 De Vecchi E, Bortolin M, Signori V, Romano CL and Drago L: Treatment With Dithiothreitol Improves Bacterial Recovery From Tissue Samples in Osteoarticular and Joint Infections. J Arthroplasty 31(12): 2867-2870, 2016.

18 Schäfer P, Fink B, Sandow D, Margull A, Berger I and Frommelt L: Prolonged Bacterial Culture to Identify Late Periprosthetic Joint Infection: A Promising Strategy. Clin Infect Dis 47(11): 1403-1409, 2008.
Received May 23, 2017

Revised June 9, 2017

Accepted June 12, 2017 